\title{
Percepción sobre el profesionalismo médico en estudiantes del último año de medicina en una universidad peruana.
}

\author{
Perception of medical professionalism in students of the last year of their career in a Peruvian university.
}

\author{
Ray Ticse ${ }^{1, a}$, Néstor E. Vásquez ${ }^{2, b}$, Hernán A Cárcamo ${ }^{2, b}$
}

\section{RESUMEN}

Objetivo: Describir conceptos, experiencias y perspectivas que alumnos del último año de medicina en una universidad peruana tienen respecto al Profesionalismo Médico. Material y Métodos: Se aplicó una encuesta a alumnos que cursaban la rotación clínica final de su último $\left(7^{\circ}\right)$ año de estudios en la Facultad de Medicina de la Universidad Peruana Cayetano Heredia, durante el año 2015. Resultados: El porcentaje de respuestas fue 90,5\%. Las tres respuestas más comunes fueron ética $(51,2 \%)$, buena comunicación médico-paciente (43\%) y conocimientos (41,9\%). El 81,4 \% de los estudiantes estuvo "de acuerdo" o "muy de acuerdo" con el hecho de que profesionalismo puede ser aprendido y enseñado. El contacto con modelos positivos del personal docente de la Facultad $(4,26 / 5,00)$ fue considerado el método más útil para el aprendizaje sobre profesionalismo médico, en tanto que sólo el 25,6\% consideró adecuado el número de actividades dedicadas a la enseñanza del tema. Conclusiones: Los Internos de medicina consideran a la ética, una buena relación médico-paciente, conocimiento, respeto y responsabilidad como principales atributos del profesionalismo médico. Percibieron que su concepto de profesionalismo fue más influenciado por modelos positivos que por actividades académicas formales.

PALABRAS CLAVE: Profesionalismo médico, estudiantes de medicina, ética.

\section{SUMMARY}

Objective: To describe concepts, experiences, and perspectives from senior ( $7^{\text {th }}$ Year) medical students of a Peruvian university, regarding Medical Professionalism. Material and Methods: A survey was applied to medical students that completed their Internship at the Faculty of Medicine of the Universidad Peruana Cayetano Heredia, during 2015. Results: The percentage of responses reached a 90.5\%. The three most common responses were ethics (51.2\%), a good doctor-patient relationship (43\%), and knowledge (41.9\%). Approximately eighty-one percent $(81.4 \%)$ of the students "agreed" or "strongly agreed" with the fact that professionalism can be learned and taught. The contact with positive models from the faculty $(4.26 / 5.00)$ was considered as the most useful method for learning about professionalism, while only $25.6 \%$ considered adequate the number of activities dedicated to the teaching of the topic. Conclusions: Medical interns consider ethics, good patient-physician communication, knowledge, respect and responsibility as the main attributes of medical professionalism. They perceived that their concept of professionalism was more influenced by positive faculty models than by formal academic activities.

KEYWORDS: Medical professionalism, medical students, ethics.

Unidad de Educación Médica, Facultad de Medicina, Universidad Peruana Cayetano Heredia. Lima, Perú.

Facultad de Medicina, Universidad Peruana Cayetano Heredia. Lima, Perú.

Médico especialista en Medicina Interna y Endocrinología. ORCID: https://orcid.org/0000-0002-4568-5723

b Médico cirujano 


\section{INTRODUCCIÓN}

En el tiempo reciente se ha visto afectada la confianza que tenía usualmente la sociedad sobre el profesionalismo de los médicos. Entre los factores que afectan esta confianza se consideran a cambios en los estilos de vida en la sociedad, cambios en los horarios de atención, expectativas en la carrera de medicina, influencia de la industria farmacéutica, entre otros $(1,2)$. Durante la carrera de medicina Los estudiantes se enfrentan a una gran cantidad de comportamiento no profesional dentro del hospital, muchas veces con modelos negativos, que puede afectar el clima educacional con tolerancia al maltrato a estudiantes $(3,4)$.

En países de altos ingresos económicos existen Instituciones acreditadoras de programas de educación médica, por ejemplo, el Royal College of Physicians of Canada que elaboró las CanMEDS (Canadian Medical Education Directives for Specialist), reconoce que se deben lograr las siguientes competencias: médico experto, comunicador, colaborador, gestor, consejero de salud, erudito (scholar) y profesional. El Comité de acreditación de los programas de postgrado de los Estados Unidos de América (EUA) elaboró el ACGME (Accreditation Council for Graduate Medical Education) considera estas competencias: Profesionalismo, habilidades interpersonales, conocimiento médico, cuidados del paciente, práctica basada en el contexto del sistema de salud, práctica clínica basada en el aprendizaje y la mejora continua (5). En el Perú, todavía no está implementado el profesionalismo como competencia en las carreras de medicina, es decir no se realiza la enseñanza y evaluación de manera específica ni regular.

Se recomienda que la implementación que se realice sobre profesionalismo en la malla curricular de una escuela o facultad de medicina debe basarse en la definición que hayan asumido como propia, la cual está de acuerdo a la sociedad en la cual se desarrolla. Esta definición debe ser difundida a los alumnos desde su ingreso a la carrera, y reforzada durante los siguientes años académicos (6). Al ser los estudiantes parte de la universidad y de la sociedad, es muy importante determinar su percepción sobre esta competencia. Por este motivo aplicamos una encuesta a estudiantes del último año académico de la carrera de medicina de una universidad peruana para determinar su percepción sobre el profesionalismo médico.

\section{MATERIAL Y MÉTODOS}

Este estudio es observacional, descriptivo, y transversal. La población de estudio fueron los estudiantes que egresaron de la carrera de Medicina de la Universidad Peruana Cayetano Heredia (FAMEDUPCH) de Lima-Perú, en el año 2015.

El diseño de la encuesta se basó en una encuesta aplicada en publicaciones previas sobre percepción de profesionalismo en estudiantes de medicina (7-10). Se realizó la validación de idioma con la participación de dos docentes peruanos cuya lengua materna era castellano y residían en EUA. Se realizó la validez de contenido de esta encuesta mediante un comité de validación conformado por seis docentes especialistas en educación médica.

La encuesta constó de 12 preguntas; 3 de respuesta abierta y 9 de opción múltiple según escala de Likert. $\mathrm{Se}$ categorizaron las respuestas a las preguntas abiertas según sinonimia por dos investigadores independientes. Luego se realizó la revisión de discrepancias para llegar a un consenso.

Criterios de inclusión: estudiantes que egresaron de la FAMED-UPCH el año 2015; de exclusión: no brindar su consentimiento o no poder ubicarlos. La encuesta fue resuelta de manera individual por cada alumno; de no haber sido posible resolver la encuesta de manera escrita, se acordó con cada encuestado enviar la encuesta vía email institucional. El protocolo de investigación fue revisado y aprobado por el Comité Institucional de Ética de Investigación de la UPCH. Los nombres de los participantes fueron reemplazados por códigos para su posterior análisis, solo los investigadores principales tuvieron acceso a la información.

\section{RESULTADOS}

El porcentaje de alumnos que respondieron la encuesta fue de 90,5\% (86/95), de los cuales el $72 \%(62 / 86)$ resolvieron por escrito. El 28\% (24/86) restante lo resolvió de manera electrónica. No se pudo contactar a 6 internos y 2 rechazaron participar del estudio. Se excluyó a 1 sujeto del estudio en base a los criterios de exclusión propuestos.

La edad promedio fue de 24,86 años. No se encontraron diferencias significativas respecto al sexo (46 hombres y 40 mujeres). 
Se registraron un total de 356 atributos para definir el profesionalismo médico. Las tres respuestas más comunes fueron ética (44 respuestas, 51,2\%), buena relación médico paciente (37 respuestas, 43\%) y conocimientos (36 respuestas, 41,9\%). Los términos con un porcentaje del total de respuestas menor del $2 \%$ fueron agrupadas en la categoría de "Otros". Cuatro participantes dejaron la respuesta en blanco o escribieron "No sé" (gráfico 1).

Por otro lado, el contacto con modelos positivos de la facultad $(4,26 / 5,00)$, el contacto con pacientes $(4,16 / 5,00)$ y las familias/amigos de los estudiantes $(3,99 / 5,00)$ fueron calificadas como las más útiles para el aprendizaje sobre profesionalismo. Las clases o seminarios, videos o películas y libros o literatura obtuvieron el puntaje más bajo en utilidad (tabla 1).

El 81,4 \% (70/86) de los estudiantes estuvo de acuerdo o muy de acuerdo con el hecho que el profesionalismo puede ser aprendido y enseñado. En cuanto a la calificación para el número de actividades relacionadas con el aprendizaje del profesionalismo, el $69,7 \%(60 / 86)$ lo consideraron normal o deficiente; y al referirse a la calidad de dichas actividades, $75,6 \%(65 / 86)$ refirieron que era adecuada o normal, $52,3 \%(45 / 86)$ se sienten cómodos o muy cómodos explicando el concepto de profesionalismo médico a un estudiante de medicina menor, y sólo el12,8\% $(11 / 86)$ presentaron algún grado de incomodidad (tabla 2).

Durante su entrenamiento en la facultad de medicina, 56 participantes $(65,1 \%)$ refirieron haber estado expuestos a situaciones profesionalmente desafiantes. Adicionalmente, 66 participantes (76,8\%) consideraron que la instrucción recibida fue de ayuda para lidiar con dichas situaciones algunas veces o de vez en cuando. El 75,5 \% de los internos encuestados consideraron que la evaluación del profesionalismo debería ser un criterio en la evaluación formal de sus calificaciones. En menor medida, el 65,1\% consideró que se debería incluir al profesionalismo como un criterio de selección para el ingreso a futuras carreras o programas académicos.

Al solicitar a los alumnos que nombren el área dentro de la organización de la facultad de medicina que lidia con temas concernientes al profesionalismo médico, las respuestas más frecuentes fueron el comité de ética (32 respuestas, 37,2\%) y la dirección de pregrado (14 respuestas, 16,3\%). Los términos mencionados dos o una vez fueron agrupados en la categoría "Otros" (8 respuestas, 9,3\%). Las respuestas en blanco fueron agrupadas con las respuestas "No sé" (32 respuestas, 37,2\%).

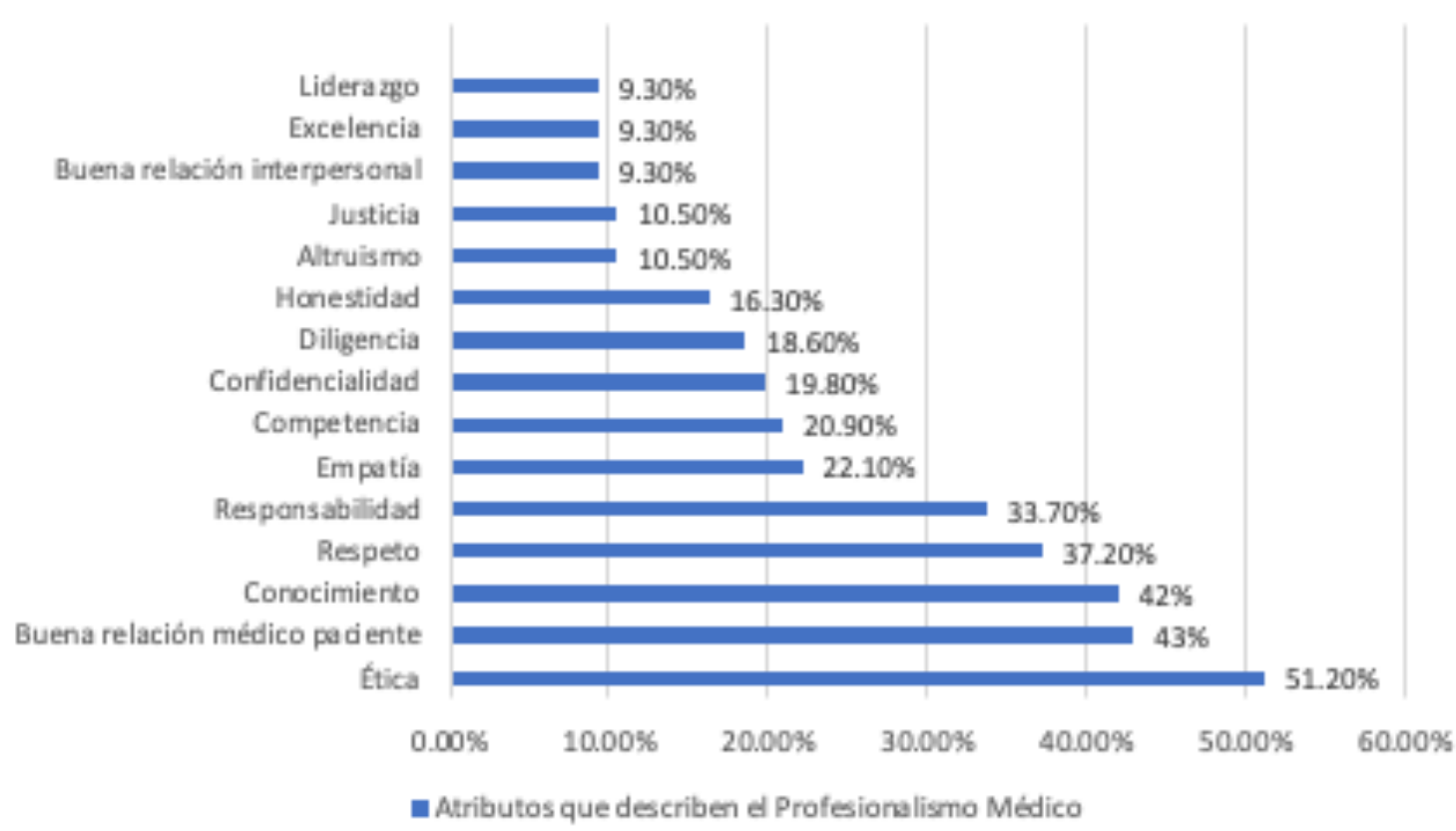

Gráfico 1. Percepción y conceptos sobre profesionalismo médico en internos de medicina de una universidad de Lima, Perú. Porcentajes de características a la pregunta abierta: ¿Qué entiende usted por Profesionalismo Médico? Enumere 5 atributos (frases /palabras/explicaciones) que lo definan. 
Tabla 1.Percepción y conceptos sobre profesionalismo médico en internos de medicina de una universidad de Lima, Perú. Durante los años que ha estudiado en la carrera de medicina de la UPCH, enumere cada ítem del 1 al 5 (siendo 5 muy útil y 1 poco/no útil) según cuanto lo han ayudado a aprender sobre Profesionalismo.

\begin{tabular}{lc}
\hline & $\begin{array}{c}\text { Promedio } \\
(\mathbf{0}-\mathbf{5})\end{array}$ \\
\hline Contacto con modelos positivos: médicos - profesores & 4,26 \\
Contacto con pacientes y sus familias & 4,16 \\
Propia familia y/o amigos & 3,99 \\
Contactos con modelos negativos: médicos - profesores & 3,31 \\
Clases o seminarios & 2,94 \\
Libros o literatura & 2,76 \\
Videos, películas o shows de TV & 2,60 \\
\hline
\end{tabular}

Tabla 2. Percepción y conceptos sobre profesionalismo médico en internos de medicina de una universidad de Lima, Perú.

\begin{tabular}{|c|c|c|c|c|c|}
\hline & n (\%) & n (\%) & n (\%) & n (\%) & n (\%) \\
\hline \multirow{2}{*}{$\begin{array}{l}\text { Respecto a la frase "El profesionalismo puede ser } \\
\text { aprendido y enseñado", usted considera que está: }\end{array}$} & $\begin{array}{l}\text { Muy de } \\
\text { acuerdo }\end{array}$ & $\begin{array}{c}\text { De } \\
\text { acuerdo }\end{array}$ & Neutral & $\begin{array}{c}\text { En } \\
\text { desacuerdo }\end{array}$ & $\begin{array}{c}\text { Muy en } \\
\text { desacuerdo }\end{array}$ \\
\hline & $18(20,9)$ & $52(60,5)$ & $10(11,6)$ & $4(4,7)$ & $2(2,3)$ \\
\hline \multirow{2}{*}{$\begin{array}{l}\text { ¿Cómo calificaría usted el número de actividades } \\
\text { relacionadas a la enseñanza del Profesionalismo } \\
\text { brindada por la Facultad? }\end{array}$} & $\begin{array}{c}\text { Muy } \\
\text { adecuada }\end{array}$ & Adecuada & Normal & Deficiente & $\begin{array}{c}\text { Muy } \\
\text { deficiente }\end{array}$ \\
\hline & $3(3,5)$ & $19(22,1)$ & $34(39,5)$ & $26(30,2)$ & $4(4,7)$ \\
\hline \multirow{2}{*}{$\begin{array}{l}\text { En base a lo que le han enseñado sobre } \\
\text { Profesionalismo Médico, ¿cómo calificaría usted la } \\
\text { calidad de enseñanza sobre Profesionalismo brindada } \\
\text { por la Facultad? }\end{array}$} & $\begin{array}{c}\text { Muy } \\
\text { adecuada }\end{array}$ & Adecuada & Normal & Deficiente & $\begin{array}{c}\text { Muy } \\
\text { deficiente }\end{array}$ \\
\hline & $4(4,7)$ & $28(32,6)$ & $37(43,1)$ & $14(16,3)$ & $3(3,5)$ \\
\hline \multirow{2}{*}{$\begin{array}{l}\text { ¿Qué tan cómodo se siente usted al explicar el } \\
\text { concepto de Profesionalismo Médico a un estudiante de } \\
\text { medicina menor año? }\end{array}$} & $\begin{array}{l}\text { Muy } \\
\text { cómodo }\end{array}$ & Cómodo & $\begin{array}{l}\text { Me es } \\
\text { indistinto }\end{array}$ & Incómodo & $\begin{array}{c}\text { Muy } \\
\text { incómodo }\end{array}$ \\
\hline & $6(6,9)$ & $39(45,3)$ & $30(34,9)$ & $10(11,6)$ & $1(1,2)$ \\
\hline \multirow[t]{2}{*}{$\begin{array}{l}\text { ¿Con qué frecuencia ha estado expuesto a situaciones } \\
\text { profesionalmente desafiantes (conductas ó conflictos) } \\
\text { durante su entrenamiento en la Facultad de Medicina? }\end{array}$} & Siempre & $\begin{array}{l}\text { Algunas } \\
\text { veces }\end{array}$ & $\begin{array}{l}\text { De vez en } \\
\text { cuando }\end{array}$ & Raramente & Nunca \\
\hline & $10(11,6)$ & $46(53,5)$ & $27(31,4)$ & $3(3,5)$ & 0 \\
\hline \multirow{2}{*}{$\begin{array}{l}\text { ¿Qué tanto le ha ayudado la instrucción recibida en } \\
\text { la Facultad de Medicina para lidiar con situaciones } \\
\text { profesionalmente desafiantes (conductas o conflictos)? }\end{array}$} & Siempre & $\begin{array}{l}\text { Casi } \\
\text { siempre }\end{array}$ & $\begin{array}{l}\text { De vez en } \\
\text { cuando }\end{array}$ & Raramente & Nunca \\
\hline & $4(4,7)$ & $25(29,1)$ & $41(47,7)$ & $15(17,4)$ & $1(1,2)$ \\
\hline \multirow[t]{2}{*}{$\begin{array}{l}\text { ¿Cree usted que la evaluación del profesionalismo } \\
\text { debería ser un criterio en su evaluación oficial/formal } \\
\text { en sus calificaciones? }\end{array}$} & $\begin{array}{l}\text { Muy de } \\
\text { acuerdo }\end{array}$ & $\begin{array}{l}\text { De } \\
\text { acuerdo }\end{array}$ & Neutral & $\begin{array}{c}\text { En } \\
\text { desacuerdo }\end{array}$ & $\begin{array}{l}\text { Muy en } \\
\text { desacuerdo }\end{array}$ \\
\hline & $26(30,2)$ & $39(45,3)$ & $17(19,8)$ & $1(1,2)$ & $3(3,5)$ \\
\hline \multirow{2}{*}{$\begin{array}{l}\text { ¿Cree usted que el Profesionalismo debería ser un } \\
\text { criterio de selección de ingreso a futuras carreras o } \\
\text { programas académicos? }\end{array}$} & $\begin{array}{l}\text { Muy de } \\
\text { acuerdo }\end{array}$ & $\begin{array}{c}\text { De } \\
\text { acuerdo }\end{array}$ & Neutral & $\begin{array}{c}\text { En } \\
\text { desacuerdo }\end{array}$ & $\begin{array}{l}\text { Muy en } \\
\text { desacuerdo }\end{array}$ \\
\hline & $32(37,2)$ & $24(27,9)$ & $21(24,4)$ & $7(8,1)$ & $2(2,3)$ \\
\hline
\end{tabular}


Percepción sobre el profesionalismo médico en estudiantes del último año de medicina en una universidad peruana.

Las respuestas más comunes de los participantes al pedir que nombren la persona u oficina para la discusión en caso se encuentren con una situación profesionalmente desafiante fueron un asistente de confianza (32 respuestas, $37,2 \%$ ), el coordinador de la rotación o curso (21 respuestas, $24,6 \%)$ y el director de pregrado (11 respuestas, 12,8\%). El resto de términos fueron agrupados en la categoría "Otros" (10 respuestas, 11,6\%). Las respuestas en blanco fueron agrupadas con las respuestas "No sé" (12 respuestas, $14 \%)$.

\section{DISCUSIÓN}

Los atributos señalados con mayor frecuencia por los estudiantes como ética, buena relación médico paciente, conocimiento, respeto y responsabilidad, coinciden en gran medida por o señalado en el "Physician Charter", carta fue elaborada en el año 2002, por el American Board of Internal Medicine (ABIM), American College of Physicians (ACP) y el European Federation of Internal Medicine (EFIM), donde definen el profesionalismo médico como "...la base del contrato de la medicina con la sociedad." . Proporciona un marco ético, educativo y práctico para guiar a los médicos en la práctica de la medicina y sus relaciones con pacientes, colegas y la sociedad. Es aplicable a diferentes culturas y sistemas políticos. Se establecen tres principios fundamentales: Primacía del bienestar del paciente, Autonomía del paciente y justicia social $(11,12)$.

Desde los inicios de la medicina la sociedad ha relacionado a la ética como un atributo que todo médico debe lograr, este atributo también con frecuencia fue asociado en encuestas aplicadas en otras escuelas o facultades de medicina, que incluso, tienen incorporado el profesionalismo como competencia con estrategias específicas de enseñanza y evaluación (1315). Además, otro factor que explique este hallazgo puede ser el hecho de los estudiantes recibieron cursos sobre ética dentro de la malla curricular y relacionen fuertemente el concepto de profesionalismo a este atributo, sin embargo, este resulta insuficiente para lograr desarrollar el profesionalismo como competencia.

Es necesario que cada institución genere su propia definición de profesionalismo, acorde a su contexto social y comunidad universitaria. Esta definición debe ser impartida a los estudiantes desde su ingreso a la carrera de medicina. La percepción de profesionalismo está influenciada por aspectos culturales o de contexto de cada región, por ejemplo, en sociedades consideradas como conservadoras se valora el respeto por el orden social, tradición y la seguridad familiar. En otras, se resaltan cualidades como puntualidad, respeto y adecuada imagen personal $(7,8,16)$. En este estudio los estudiantes encuestados declararon el contacto con los pacientes, sus familiares, su propia familia y amigos influyeron en sus conceptos de profesionalismo. Sin embargo, la percepción de los estudiantes puede cambiar con el tiempo. En el pregrado lo relacionan fuertemente con la ética, por el contrario, los médicos residentes suelen adoptar una visión más integral, vinculada a aspectos que se presentan en la práctica clínica, como son conocimiento, respeto y empatía hacia los pacientes $(9,17)$. Sin embargo, lamentablemente algunas actitudes positivas se pueden deteriorar con el tiempo por lo que se debe reforzar durante la carrera en pregrado y posgrado $(18,19)$.

La relación médico paciente también fue señalada como característica relevante. Esta habilidad forma parte de una competencia más amplia que corresponde a la comunicación clínica; implica la comunicación con sus pares, con miembros del equipo de salud, y relaciones interprofesionales. Requiere de habilidades como la escucha activa, empatía, etc. Los pacientes valoran bastante esta habilidad y lo cual impacta en su percepción tanto de la comunicación como del profesionalismo de los médicos $(20,21)$. La FAMEDUPCH ha implementado la comunicación como competencia clínica, tanto en el pregrado como en el posgrado, con la aplicación de cursos de entrenamiento y la evaluación formativa y sumátiva con instrumentos, como por ejemplo, el ECOE (Examen Clínico Objetivo Estructurado) (22,23). Queda claro que el profesionalismo y la comunicación son competencias que requieren el desarrollo de habilidades en común, muchas veces los escenarios que se aplican en las estaciones de ECOE para evaluarlas son las mismas, sin embargo, los ítems que se evalúan son diferentes (24).

Los estudiantes percibieron que lo más influyente en su aprendizaje fue lo brindado por sus experiencias durante la carrera de medicina, como es el contacto con modelos positivos de los médicos y profesores; y otros estudiantes. Incluso, los encuestados perciben que pueden explicar el concepto de profesionalismo a estudiantes de años académicos menores, esto puede deberse a que se desempeñan como docentes de estos 
$(25,26)$. Es lo que se denomina "curriculum oculto" o informal; las reglas no escritas, los hábitos, los códigos y los rituales presentes en la profesión médica se experimentan implícitamente casi como un rito de iniciación. Cumplir con estas puede ser una estrategia de supervivencia exitosa. Los roles que ejercen los modelos influyen en el clima educacional o liderazgo y el aprendizaje por experiencia $(9,12)$.

La mayoría de los encuestados estuvo de acuerdo que debería ser parte de sus calificaciones, incluso, que debería ser criterio de selección para la admisión a la FAMED-UPCH. Sin embargo, sólo la cuarta parte percibió como adecuada la calidad de la enseñanza sobre profesionalismo. Para que se logre consolidar al profesionalismo como competencia se requiere consolidar un curriculum formal (27-29). Este debe proveer de diversos tipos de experiencias como la discusión de casos, rondas clínicas, cursos de ética, clases de humanismo, elaboración de ensayos a propósitos de incidentes, discusión de aspectos legales, implementación de seminarios anuales "Journal Club" sobre profesionalismo, entrenamiento en habilidades de comunicación, liderazgo y reflexión, incorporación de aspectos de profesionalismo en las discusiones sobre morbilidad y mortalidad, desarrollo de habilidades mediante la simulación clínica, entre otras alternativas $(12,30)$.

Los estudiantes declararon que las situaciones profesionalmente desafiantes fueron frecuentes. Estas situaciones son más frecuentes cuando realizan prácticas clínicas, donde tienen contacto con pacientes, familiares de pacientes, trabajo en equipo con sus pares e interprofesional $(13,14)$. La falta de información explicita sobre este tema hace que generalmente no sepan cómo proceder en estas situaciones y no se llegue a denunciar. Esto es especialmente perjudicial cuando los estudiantes se desenvuelven en climas de educación en donde predominen los modelos negativos, haciendo que estos se perpetúen, incluso pueden formar parte del curriculum oculto $(3,4,18,30)$. Una de las fortalezas de este estudio es que permite conocer la percepción de los alumnos del último año de la carrera sobre el profesionalismo como competencia médica, esto permitirá a la facultad poder implementar modificaciones en los contenidos de la malla curricular, e implementar estrategias para conseguir que logren el concepto como parte de sus logros de aprendizaje. De hecho, en el 2019, posterior a este estudio, la FAMEDUPCH incorporó al profesionalismo como una de las competencias específicas de la carrera: "Actuar con profesionalismo y compromiso social en los campos en los que desarrolla su actividad, actualizándose y practicando autoaprendizaje y responsabilizándose de su bienestar personal (Conducta profesional)".

Entre las limitaciones de este estudio consideramos el no haber realizado la evaluación psicométrica de la encuesta, sólo encuestar a estudiantes del último año de carrera de medicina, lo que no permite evaluar la evolución ni los cambios que se dan a lo largo de la carrera. Además, al utilizar una encuesta por escrito se pudo haber limitado la oportunidad de explayarse y desarrollar las ideas u opiniones que los alumnos pudieron haber tenido respecto al profesionalismo.

En conclusión, los estudiantes encuestados del último año académico de la carrera de medicina de la FAMEDUPCH consideraron con mayor frecuencia a la ética, la buena relación médico paciente, conocimientos, respeto y responsabilidad, como atributos que describen el profesionalismo. Percibieron que más influyó los modelos positivos que las actividades académicas en su concepto de profesionalismo. Se recomienda modificar el curiculum formal para incrementar la cantidad y calidad de las actividades académicas respecto al profesionalismo.

El informe preliminar de este estudio fue presentado en el V Congreso Internacional de Educación Médica de la Asociación Peruana de Facultades de Medicina, CIEM2019.

\section{Correspondencia:}

Ray Willy Ticse Aguirre.

Unidad de Educación Médica (UEM), Facultad de Medicina Alberto Hurtado, Universidad Peruana Cayetano Heredia,

Av. Honorio Delgado 430, San Martín de Porres, Lima, Perú.

Correo electrónico: ray.ticse@upch.pe

\section{REFERENCIAS BIBLIOGRÁFICAS}

1. Coulehan J. Today's professionalism: Engaging the mind but not the heart. Acad Med. 2005; 80:892-8.

2. De Ferrari A, Gentille C, Davalos L, Huayanay L, Malaga G. Attitudes and relationship between physicians and the pharmaceutical industry in a public general hospital in Lima, Peru. PLoS ONE. 2014; 9(6): e100114. doi:10.1371/journal. pone. 0100114

3. Munayco-Guillén F, Cámara-Reyes A, MuñozTafur LJ, Arroyo-Hernández H, Mejia CR, Lem-Arce 
Percepción sobre el profesionalismo médico en estudiantes del último año de medicina en una universidad peruana.

F, Miranda-Soberón UE. Características del maltrato hacia estudiantes de medicina de una universidad pública del Perú. Rev Peru Med Exp Salud Publica. 2016; 33(1): 58-66. doi: 10.17843/rpmesp. 2016.331.2008.

4. Sierra A, Zárate J, Mejia CR. Maltrato asociado al acoso laboral a internos de Medicina en hospitales peruanos. Educación Médica Superior. 2019;33 (4):e1720.

5. Ticse R. El Examen Clínico Objetivo Estructurado (ECOE) en la evaluación de competencias de comunicación y profesionalismo en los programas de especialización en Medicina. Rev Med Hered. 2017; 28:192-199.

6. Cruess SR, Cruess RL, Steinert Y. Teaching professionalism across cultural and national borders: Lessons learned from an AMEE workshop. Med Teach. 2010; 32(5):371-374.

7. Chandratilake M, McAleer S, Gibson J. Cultural similarities and differences in medical professionalism: a multi-region study. Med Educ. 2012; 46(3):257-266.

8. Al-Abdulrazzaq D, Al-Fadhli A, Arshad A. Advanced medical students' experiences and views on professionalism at Kuwait University. BMC Medical Education. 2014; 14:150.

9. Brownell AK, Cote L. Senior residents' views on the meaning of professionalism and how they learn about it. Acad Med. 2001; 76(7):734-737.

10. Adkoli BV, Al-Umran KU, Al-Sheikh M, Deepak KK, Al-Rubaish AM. Medical students' perception of professionalism: a qualitative study from Saudi Arabia. Med Teach. 2011; 33(10):840-845.

11. ABIM Foundation; ACP-ASIM Foundation; European Federation of Internal Medicine. Medical professionalism in the new millennium: A physician charter. Ann Intern Med. 2002; 136:243-246.

12. O'Sullivan H, van-Mook W, Fewtrell R, Wass V. Integrating professionalism into the curriculum: AMEE Guide No. 61, Medical Teacher. 2012; 34(2): e64-e77, DOI: 10.3109/0142159X.2012.655610

13. Chandratilake M, McAleer S, Gibson J. Cultural similarities and differences in medical professionalism: a multi-region study. Med Educ. 2012; 46(3):257-266.

14. Swick HM, Szenas P, Danoff D, Whitcomb ME. Teaching professionalism in undergraduate medical education. JAMA. 1999; 282(9):830-832.

15. Jiang S, Yan Z, Xie X, Tang W, Lu F, He J. Initial knowledge of medical professionalism among Chinese medical students. Med Teach. 2010; 32(12):961-970.

16. Van De Camp K, Vernooij-Dassen MJFJ, Grol RPTM, Bottema BJAM. How to conceptualize professionalism: a qualitative study. Med Teach. 2004; 26:696-702.
17. Mayo GV, Quijano EG, Ponce DA, Ticse R. Utilización de la Escala de Empatía Médica de Jefferson en Residentes que realizan su especialización en un hospital peruano. Rev Neuropsiquiatr. 2019; 82(2):131-140. DOI: 10.20453/rnp.v82i2.3540

18. Salam A. A pilot study on professionalism of future medical professionals in Universiti Kebangsaan Malaysia (UKM) Medical Centre. Procedia. 2012; 60:534-540.

19. Brown D, Ferrill MJ, Lloyd L. The taxonomy of professionalism: reframing the academic pursuit of professional development. American Journal of Pharmaceutical Education 2009; 73(4): 68.

20. Guillén-López OB, Olascoaga-Mesía AC. Habilidad de los médicos residentes para comunicar malas noticias. Rev Soc Peru Med Interna. 2017; 30 (2):78-81.

21. Quispe RC, Muñoz EM, Cadillo AV, Ticse R. Perception of medical communication skills during the clinical interview in a Peruvian teaching hospital: validation and application of an assessment tool. Medwave. 2019;19(9):e7705.

22. Lozano EA, Tafur KB, OlascoagaAC. Implementación de un curso de comunicación para médicos residentes de primer año; V Congreso Internacional de Educación Médica - CIEM2019. Lima; Asociación Peruana de Facultades de Medicina; 2019. p.25.

23. Ticse R, Cuba S, Nestares J, Aguilera L. Efecto de un entrenamiento corto en habilidades de comunicación con el método CICAA (conectar, identificar, comprender, acordar y ayudar), en médicos residentes de una universidad peruana. Rev Med Hered. 2019; 30:148-156.

24. Dwyer T, Takahashi SG, Hynes MK, et al. How to assess communication, professionalism, collaboration and the other intrinsic CanMEDS roles in orthopedic residents: use of an objective structured clinical examination (OSCE). Can J Surg. 2014; 57(4):230-6.

25. Harden RM, Laidlaw JM. Essential Skills for a Medical Teacher: An introduction to teaching and learning in medicine. New York: Elsevier; 2012.

26. Chachaima- Mar JE, Ticse R. El estudiante de medicina como profesor en el Perú: un rol importante pero olvidado. Educ Med. 2020; 21(6): 410-411. DOI: $10.1016 /$ j.edumed.2019.07.003

27. Nishigori H, Otani T, Plint S, Uchino M, Ban N. I came, I saw, I reflected: A qualitative study in to learning outcomes of international electives for Japanese and British medical students. Med Teach. 2009; 31: e196-e201.

28. Baldwin DC, Daugherty SR, Rowley BD. Unethical and unprofessional conduct observed by residents during their first year of training, Academic Medicine. 1998; 73(11): 1195-1200.

29. Feudtner C, Christakis DA, Christakis NA. Do clinical clerks suffer ethical erosion? Students' perceptions 
Ticse R, et al.

of their ethical environment and personal development. Academic Medicine. 1994; 69:670-9.

30. Al-Eraky MM. Twelve Tips for teaching medical professionalism at all levels of medical education,
Medical Teacher. 2015; 37(11): 1018-1025. DOI: 10.3109/0142159X.2015.1020288

Recibido: 03/06/2020

Aceptado: 15/02/2021 